\title{
ON SPECIALIZATIONS OF CURVES. I
}

BY

A. NOBILE

\begin{abstract}
The following is proved: Given a family of projective reduced curves $X \rightarrow T$ ( $T$ irreducible), if $X_{t}$ (the general curve) is integral and $X_{0}$ is a special curve (having irreducible components $\left.X_{1}, \ldots, X_{r}\right)$, then $\sum_{i=i}^{r} g_{i}\left(X_{i}\right) \leqslant g\left(X_{t}\right)$, where $g(Z)$ = geometric genus of $Z$. Conversely, if $A$ is a reduced plane projective curve, of degree $n$ with irreducible components $X_{1}, \ldots, X_{r}$, and $g$ satisfies $\sum_{i=1}^{r} g_{i}\left(X_{i}\right) \leqslant g$ $\leqslant \frac{1}{2}(n-1)(n-2)$, then a family of plane curves $X \rightarrow T$ (with $T$ integral) exists, where for some $t_{0} \in T, X_{t_{0}}=Z$ and for $t$ generic, $X_{t}$ is integral and has only nodes as singularities. Results of this type appear in an old paper by $G$. Albanese, but the exposition is rather obscure.
\end{abstract}

In 1928 Giacomo Albanese published a beautiful paper (cf. [1]), where he presents some very interesting results on families of plane curves. In it, he gives necessary and sufficient conditions in order that a plane curve $B$ (not necessarily reduced, i.e., an effective divisor in $\mathbf{P}^{2}, B=k_{1} B_{1}+\cdots+k_{s} B_{s}$, where $B_{1}, \ldots, B_{s}$ are the irreducible components of $B_{\text {red }}$ ) could be a specialization of an integral curve $A \subset \mathbf{P}^{2}$ (conditions in terms of the geometric genera of $A$ and $B_{i}, i=1, \ldots, s$, as well as the coefficients $k_{i}$ ). Unfortunately, even for the standards of that period, the paper is rather obscurely written. Perhaps I should quote Zariski's commment in [12, p. 216] “... but the conclusions... cannot be regarded as final in view of the extreme delicacy of the arguments involved". In spite of the many years that have gone by, apparently little or nothing appears in the modern literature in this direction. Of course, one has some deep results on degeneration of smooth curves into curves with certain types of nodes (stable curves, cf. [2]), but this theory is, probably, of a quite different nature.

In this paper, I make a modest contribution to the theory, namely I give a modern (and, I hope, rigorous) presentation of some of the results claimed by Albanese. These have to do with families of reduced curves, i.e., in the notation just introduced, in the case where $k_{i}=1$ for all $i$. The precise statements of the main results are in $\S 1$, namely Theorems (1.2) (on how the genus must behave, given a family) and (1.4) (on existence of a family or specialization, when certain numerical conditions are satisfied).

The proof of Theorem (1.2) given here is entirely different from Albanese's. The proof of (1.4) is very similar to his, actually it is his proof, with the verification of a number of details added. Curiously enough, to verify some details of this 1928 proof,

Received by the editors April 19, 1983.

1980 Mathematics Subject Classification. Primary 14H10, 14H20; Secondary 14H99.

Key words and phrases. Family of curves, genus, nodal curve, assigned singularities. 
one has to use some results from the very recent paper [10] by O. Zariski. Albanese's proof, which is a masterpiece of geometric imagination, makes a clever use of Severi's variety of curves with nodes [8, Anhang F] (fortunately, of parts of Severi's theory that can be verified, cf. [7]). One is tempted to prove the existence Theorem (1.4) by means of deformation theory, but that seems difficult because of obstructions. For instance, if $C$ is a reduced curve in $\mathbf{P}^{2}, J$ its jacobian ideal, then the fundamental sequence relating local deformations of the singularities and first-order deformations of $C$ (inside $\mathbf{P}^{2}$ ) is

$$
H^{0}\left(C, N_{C}\right) \stackrel{\alpha}{\rightarrow} H^{0}\left(C, N_{C} \otimes \mathfrak{E}_{S}\right) \stackrel{\beta}{\rightarrow} H^{1}\left(C, J \otimes N_{C}\right) \rightarrow 0
$$

where $N_{C}$ is the normal bundle; note $H^{0}\left(C, N_{C} \otimes \mathcal{E}_{S}\right) \approx \oplus_{p \in S} T_{C, p}^{1}$, $S$ being the singular set of $C$. But $H^{1}\left(C, J \otimes N_{C}\right)$ could be nonzero, so one should carefully study $\beta$, which probably is not easy, if the singularities of $C$ are complicated.

In $\S 0$ some preliminaries on notation and auxiliary concepts are presented, in $\S 1$ the main statements are given. The proof of Theorem (1.2) is in $\$ 2$. The proof of (1.3) is in $\S \S 4$ and 5 (in $\S 4$ an auxiliary theorem, namely, Theorem (1.4), interesting in itself, is presented). In $\$ 3$, some necessary concepts are reviewed. In particular, for the reader's convenience, a summary of facts from "Anhang F" that we need is given.

As pointed out before, more general results than those discussed here are claimed in [1]. There, the curve $B$ is not assumed to be reduced. Also, some geometric properties of the singularities of $B$, which are "limits" of the nodes of the general $A$, are claimed.

I intend to discuss some of these finer items in a future article.

\section{Preliminaries.}

(0.1) The basic terminology is taken from [4], with some minor changes to be explained next. However, we need only the more elementary aspects of the theory of schemes, since we shall always work with schemes over a fixed algebraically closed field $k$.

(0.2) More precisely, we work in the category $\mathbb{C} S(k)$ of algebraic schemes of finite type over a field $k$, algebraically closed and of characteristic zero. An algebraic variety is a reduced scheme in $Q S(k)$ (not necessarily irreducible). If $Z$ is an algebraic variety, $\operatorname{Sing}(Z)$ is the singular locus of $Z$. We write $\mathbf{P}^{m}$ to denote the $m$-dimensional projective space over $k$.

(0.3) If $T$ is an integral scheme (in $\mathcal{Q S}(k)$ ), the term "a (or the) general (or generic) point of $T$ " will mean a closed point, taken from a suitable open, nonempty set in $T$, which will be either clear from the context or explicitly specified. This is done trying to make the presentation more geometric. We believe that the interested reader can easily translate any argument where this convention is used into one where the expression "generic point" is used in the strict sense of the theory of schemes.

(0.4) A curve is an algebraic variety of pure dimension one. A plane curve is a closed subscheme of $\mathbf{P}^{2}$ which is a curve. A nodal curve is a reduced plane curve, 
such that all its singular points are ordinary double points or nodes. The terms "geometric genus" and "arithmetic genus" of a reduced curve $C$ are used as in [4], and denoted by $g(C)$ and $p_{a}(C)$, respectively (if $C$ has irreducible components $C_{1}, \ldots, C_{r}$, then $g(C)$ means $\left.\sum_{i=1}^{r} g\left(C_{i}\right)\right)$.

If $p \in C$ (a reduced curve), its " $\delta$ invariant" $\delta(p, C)$ is the length of the $\Theta_{C, p}$-module $\bar{\theta}_{C, p} / \hat{\theta}_{C, p}$, where a bar indicates normalization.

(0.5) Recall the "genus formula". Given a connected, reduced curve $Z$, then

$$
p_{a}(Z)=g(Z)+\sum_{p \in Z} \delta(p, Z)-r+1
$$

where $r$ is the number of irreducible components of $Z$.

(0.6) A family of curves is a flat morphism $f: X \rightarrow T$ (in $Q \mathcal{S}(k)$ ), such that for each closed point $t \in T, f^{-1}(t)$ is a curve. The family is (a) proper if $f$ is a proper morphism, and (b) of reduced curves if $f^{-1}(t)$ is reduced for all $t \in T$. A family of plane curves (parametrized by $T$ ) is an effective relative Cartier divisor $\mathscr{D} \subset \mathbf{P}^{2} \times T$ (then if $f: \mathscr{D} \rightarrow T$ is induced by the second projection, this is a family in the sense just introduced).

Sometimes we may talk about the "generic" genus of a family of curves. Precisely, we have

(0.7) LEMMA. Let $f: X \rightarrow T$ be a proper family of reduced curves, parametrized by an irreducible scheme $T$, such that the general curve is irreducible. Then, there is a nonnegative integer $p$ and an open $U \neq \varnothing$ of $T$, such that for each $t \in T, X_{t}$ is an integral curve of geometric genus $p$.

Proof. By pulling-back over $T_{\text {red }}$, and then to the open of nonsingular points of $T_{\text {red }}$, we may assume $T$ integral and smooth. Then, since $f$ is flat and the generic fiber is integral, it follows that $X$ is integral. Take the normalization $q: Z \rightarrow X$ of $X$, let $\pi=f \cdot q$. Then, $\operatorname{dim} Z=\operatorname{dim} T+1, \operatorname{Sing}(Z)=S$ has codimension $\leqslant 2$, and $\pi(S)$ has codimension $\leqslant 1$. Apply Corollary 10.7 of $[4$, p. 272] to the induced morphism: $\pi^{-1}\left(T-S^{\prime}\right) \rightarrow T-S^{\prime}$ with $S^{\prime}=\pi(S)$. It follows for an open $V$ of $T$, if $t \in V$ then $h^{-1}(t)=\pi^{-1}(t)=Z_{t}$ is smooth and for a suitable open $U \subset V, Z_{t}$ is the normalization of $X_{t}$ (for the latter, apply again Corollary 10.7 of [4] to $X$-Sing $(X) \rightarrow T$ ). Thus, for $t \in U, g\left(X_{t}\right)=p_{a}\left(Z_{t}\right)$. But since we may assume that $\pi^{-1}(U) \rightarrow U$ is flat (by generic flatness), $p_{a}\left(Z_{t}\right)$ is constant for $t \in U$.

(0.8) Definition. Given a proper family of reduced curves $f: X \rightarrow T$, with $T$ irreducible, the generic genus of the family is $g\left(X_{t}\right)$, for $t \in \cdot U$, where $U$ is an open as that of Lemma (0.7).

\section{Statement of the main results.}

(1.1) Next, we state the main results to be proved in this article.

Throughout, we work with an algebraically closed field $k$ of characteristic zero.

(1.2) THEOREM. Let $f: X \rightarrow T$ be a proper family of reduced curves, where the general curve is irreducible, of geometric genus $g(c f .(0.7))$, and $T$ is an irreducible algebraic $k$-scheme. Let $t_{0} \in T$ be a closed point, $f^{-1}\left(t_{0}\right)=B=\cup_{i=1}^{r} B_{i}$ (where $B_{1}, \ldots, B_{r}$ are the irreducible components of $\left.B\right), g_{i}$ the geometric genus of $B_{i}, i=1, \ldots, r$. 
Then,

$$
g \geqslant \sum_{i=1}^{r} g_{i}
$$

Note that $f$ is not assumed to be a family of plane curves.

Theorem (1.2) has the following converse.

(1.3) TheOREM. Let $B$ be a projective, plane, reduced curve of degree $n$, having irreducible components $B_{1}, \ldots, B_{r}$ (the curve $B$ can have arbitrary singularities). Let $g_{i}$ be the geometric genus of $B_{i}$, and $g$ a given integer, satisfying

$$
\sum_{i=1}^{r} g_{i} \leqslant g \leqslant \frac{(n-1)(n-2)}{2}
$$

Then, there is a family of plane curves

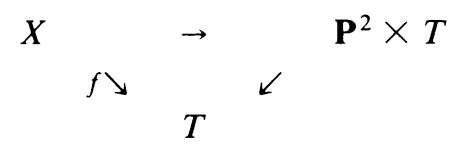

with $T$ integral, and a closed point $t_{0} \in T$, such that (a) $f^{-1}\left(t_{0}\right)=B$ and (b) the general curve is integral, of genus $g(c f .(0.4))$.

Theorem (1.2) will be a consequence of the following result.

(1.4) Theorem. Let $B$ be an integral plane curve of degree $n$ and geometric genus $p$, having arbitrary singularities. Then, there is a family of plane curves $X \stackrel{f}{\rightarrow} T, T$ integral, and $t_{0} \in T$ such that $f^{-1}\left(t_{0}\right)=B$ and the general member is an irreducible nodal curve of geometric genus $p$.

\section{Proof of Theorem (1.2).}

(2.1) Here we present a proof of Theorem (1.2). A proof of a similar result, but where the specializations are in the sense of the theory of Chow coordinates and not of flat families, can be seen in [5].

(2.2) Proof of (1.2). By pulling back over the normalization of a suitable integral curve $T_{1} \subset T$, we may assume that $T$ is a smooth, integral curve. Then it easily follows that $X$ must be an integral surface. Take the normalization $\pi: Z \rightarrow X$ of $X$. Now, $Z$ is a normal surface, hence Cohen-Macaulay. Since the composite morphism $p=f \circ \pi: Z \rightarrow T$ is equidimensional and $T$ is smooth, it follows that $p$ is flat.

We claim that $p$ defines a family of reduced curves, where, for all $t \in T, p^{-1}(t)$ is birationally equivalent to $f^{-1}(t)$ and, for $t$ generic, $f^{-1}(t)$ is smooth. To begin with, since $T$ is a smooth curve, the fibers $f^{-1}(t)$ will be Cartier divisors (defined at $\theta_{Z, z}$ by $p^{*}(t)=0, t$ a parameter of $\left.\Theta_{T, f(z)}\right)$. Since $Z$ is Cohen-Macaulay, each divisor $Z_{t}=p^{-1}(t)$ will be Cohen-Macaulay. Similarly, $X_{t}=f^{-1}(t)$ is a divisor of $X$. Consider $X_{t}$, which is reduced by assumption. In each component of $X_{t}$, we may find points $x$ such that $X_{t}$ is smooth at $x$. Since $X_{t}$ is a divisor of $X$, it follows that $X$ is smooth at $x$. From this, we may easily find an open $U$ in $X$ such that $U \cap X_{t}$ is dense in $S_{t}$ and that the normalization map $\pi$ induces an isomorphism $\pi^{-1}(U) \stackrel{\sim}{\rightarrow} U$. It 
follows that $\pi^{-1}(U) \cap Z_{t} \approx U \cap X_{t}$, hence $Z_{t}$ is "generically reduced" and, being Cohen-Macaulay, it is everywhere reduced. The isomorphism above also shows the claimed birationality statement. The assertion " $Z_{t}$ is smooth for $t$ generic in $T$ " follows from "generic smoothness" (see [4, Corollary 10.7, p. 272]), applied to the morphism $Z-p^{-1}(S) \rightarrow T-S$, where $S=p(\operatorname{Sing}(Z))$ (note $\operatorname{Sing}(Z)$ is a finite set, since $Z$ is normal).

Now, take $t$ generic in $T$, and let $Z_{0}=Z_{t_{0}}\left(t_{0}\right.$ is the given point in (1.2)). By flatness, $p_{a}\left(Z_{t}\right)=p_{a}\left(Z_{0}\right)$. But $Z_{t}$ is just the normalization of $X_{t}$, hence $p_{a}\left(Z_{t}\right)=g$ $=$ geometric genus of $X_{t}$. On the other hand, $Z_{0}$ is connected by the "connectedness principle" (cf. [4, p. 281]), hence $p_{a}\left(Z_{0}\right)=g\left(Z_{0}\right)+\Sigma_{z \in Z_{0}} \delta\left(z, Z_{0}\right)-(r-1)$, and $g\left(Z_{0}\right)=g\left(X_{0}\right)$ by birationality. But we also have $\Sigma_{z \in Z_{0}} \delta\left(z, Z_{0}\right) \geqslant(r-1)$. In fact, this is an easy consequence of the connectedness of $Z_{0}$ (e.g., see [5, p. 180]). Putting these facts together, we get $g \geqslant g\left(X_{0}\right)=\sum_{i=1}^{r} g_{i}$, as desired.

3. Review of some useful results. Here we review some known facts that are necessary to prove Theorems (1.3) and (1.4). In (3.1) and (3.2) we review facts about "Severi's variety of nodal curves." For details see [8, Anhang F] or (in a modern presentation) [7].

(3.1) (a) All plane curves of degree $n$ are parametrized by a $\mathbf{P}^{N}, N=\frac{1}{2} n(n+3)$. More precisely, this is the connected component of the Hilbert scheme of $\mathbf{P}^{2}$ corresponding to effective divisors of degree $n$. There is a universal curve $D \subset \mathbf{P}^{N} \times$ $\mathbf{P}^{2}$, having equation

$$
\sum a_{i j l} x_{0}^{i} x_{1}^{j} x_{2}^{l}=0, \quad i+j+l=n
$$

Given any family $X \rightarrow T$ of plane curves of degree $n$, there is a unique morphism $T \rightarrow \mathbf{P}^{N}$, such that $X \rightarrow T$ is isomorphic to the pull-back of the universal family.

(b) Points of $\mathbf{P}^{N}$ corresponding to curves $C$ with exactly $\delta$ nodes (i.e., $\operatorname{Sing}(C)=$ $\left\{P_{1}, \ldots, P_{\delta}\right\}$, and each $P_{i}$ is a node) form a locally closed subvariety (generally reducible) $U_{n, \delta}$, its closure will be denoted by $V_{n, \delta}^{(0)}$. Each irreducible component of $V_{n, \delta}^{(0)}$ has the same dimension, namely $N-\delta=3 n+p-1, p=$ geometric genus of any curve $C \in U_{n, \delta}$. The variety $V_{n, \delta}^{(0)}$ is the parameter space of a canonical family, $\mathscr{D}^{\prime} \rightarrow V_{n, \delta}^{(0)}$, namely the pull-back of $\mathscr{D}$ of (3.1.1) and it has the following universal property: Given a family of curves $X \rightarrow T, T$ reduced, where general fibers have exactly $\delta$ nodes, then the canonical morphism $T \rightarrow \mathbf{P}^{N}$ factors through $V_{n, \delta}^{(0)}$ (actually, it has a finer universal property, more complicated to state, which we omit because we do not need it, see [7, $\$ 3$ and 4]).

(c) The variety $V_{n, \delta}^{(0)}$ contains all curves $C$ with $\delta^{\prime} \geqslant \delta$ nodes, and no other singularity, i.e., $V_{n, \delta}^{(0)} \supset V_{n, \delta^{\prime}}^{(0)} \supset U_{n, \delta^{\prime}}$, if $\delta^{\prime} \geqslant \delta$. If $C \in U_{n, \delta^{\prime}}$, then $V_{n, \delta}^{(0)}$ has, at $C$, smooth analytic branches only; and the choice of $\delta$ among the $\delta^{\prime}$ nodes of $C$ (say, $P_{1}, \ldots, P_{\delta}$ ) determines a unique such branch $\Re$. Namely, there is exactly one branch, whose tangent space (regarded as a linear subvariety of $\mathbf{P}^{N}$ passing through $C$ ) is $\left\{D: D\right.$ is a plane curve of degree $n$ passing through $\left.P_{i}, i=1, \ldots, \delta\right\}$. Of course, this branch $\mathscr{B}$ determines a unique irreducible component of $V_{n, \delta}^{(0)}$ containing $C$. In particular, if $C$ has exactly $\delta$ nodes, $V_{n, \delta}^{(0)}$ is smooth at $C$. 
(d) Intuitively, and working over $\mathbf{C}$ (where $\mathscr{B}$ can be regarded as a "germ"), the general curve $A$ of $\mathscr{B}$ has exactly $\delta$ nodes, which approach $P_{1}, \ldots, P_{\delta}$ as $A$ approaches $C$ (see (3.2)).

(e) Given the "assignment" $P_{1}, \ldots, P_{\delta}$ on $C$ (cf. (c)), the general curve of the resulting branch (or component of $V_{n, \delta}^{(0)}$, if one prefers) will be irreducible if and only if $C-\left\{P_{1}, \ldots, P_{\delta}\right\}$ is connnected.

(3.2) Algebraically, assertion (d) about "approaching singularities" can be made precise as follows. Let $E$ be an irreducible component of $V_{n, \delta}^{(0)}, C \in \Sigma$ and $Q_{1}, \ldots, Q_{\delta}$ (not necessarily distinct) singular points of $C$. Sometimes, we would like to say: "by suitably specializing a general curve of $E$, its $\delta$ nodes approach $Q_{1}, \ldots, Q_{\delta}$ " (or "specialize into $Q_{1}, \ldots, Q_{\delta}$ "). This shall mean the following: (i) There is a morphism $\beta: T \rightarrow \Sigma$, where $T$ is a connected curve, and a point $t_{0} \in T$, such that $\beta\left(t_{0}\right)=C$, $\beta(t)$ is a nodal curve with $\delta$ nodes, for $t \neq t_{0}$. (ii) There are $\delta$ sections $\varepsilon_{i}: T \rightarrow D$, where $D \rightarrow T$ is the pull-back of the universal family over $V_{n, \delta}^{(0)}$; moreover, $\left\{\varepsilon_{1}\left(t_{0}\right), \ldots, \varepsilon_{\delta}\left(t_{0}\right)\right\}=\left\{Q_{1}, \ldots, Q_{\delta}\right\}$ and for $t \neq t_{0},\left\{\varepsilon_{1}(t), \ldots, \varepsilon_{\delta}(t)\right\}=\operatorname{Sing}\left(D_{t}\right)$.

Under these conditions, given $t_{1} \in T$, we also say that the node $\varepsilon_{i}\left(t_{1}\right)$ of $D_{t_{1}}$ specializes to $Q_{i}$. In [7, (4.11)], it is shown that always, given $C \in \Sigma$, certain singularities of $C$ will be obtained by suitably specializing a general curve $A$ of $E$ (and its nodes) in the sense just described. Actually, there the case "each $Q_{i}$ is a node" is treated, but the methods used work in general. The idea is to cover $V_{n, \delta}^{(0)}$ by a variety $W_{n, \delta}^{(0)}$, which is the closure in $\mathbf{P}^{N+2 \delta}$ of systems $\left(C, P_{1}, \ldots, P_{\delta}\right)$, with $C$ nodal, $P_{i}$ a node of $C$ for each $i$; the pull-back of the universal family $D^{\prime} \rightarrow V_{n, \delta}^{(0)}$ over $W_{n, \delta}^{(0)}$ admits $\delta$ canonical sections.

(3.3) We recall some facts from [10]. There is a concept of equivalence of singularities of locally plane curves, developed in modern terms by Zariski (see [4, p. 393 or 11]). Analytic isomorphism implies equivalence, but the converse is false. An equisingularity class (or just a "class") is an element of the set of singular points of plane curves, modulo equivalence.

In [10], Zariski proves the following:

(a) Let $f: X \rightarrow T$ be a family of plane, reduced curves (i.e., $X \subset \mathbf{P}^{2} \times T$ ) with $T$ integral. Then, there is an open set $U \subset T$, and equisingular classes $\mathcal{E}_{1}, \ldots, \mathcal{E}_{r}$, such that, for each closed point of $U, X_{t}=f^{-1}(t)$ is a curve with exactly $r$ singular points which, if suitable ordered, will be of class $\mathcal{E}_{1}, \ldots, \mathcal{E}_{r}$, respectively. Since the geometric genus of $X_{t}$ depends on the class of singular points, this gives another proof of (0.7).

(b) In particular, this applies to the case where $T \subset \mathbf{P}^{N}(N$ as in (3.1)), $T$ is integral, $f: X \rightarrow T$ is the pull-back to $T$ of the universal family in $\mathbf{P}^{2} \times \mathbf{P}^{N}$, given by (3.1.1). In this situation, we have: Let $p$ be the generic geometric genus of the family. Then, $\operatorname{dim} T \leqslant 3 n+p-1$, and the equality holds if and only if the generic member of the family is a nodal curve (and the closure of $T$ is an irreducible component of $V_{n, \delta}^{(0)}$.

(c) In the same paper (p. 224), Zariski shows the following, which will be useful in §5: Consider (notation of (3.1)) $V_{n_{1}, \delta_{1}}^{(0)}, \ldots, V_{n_{q}, \delta_{q}}^{(0)}$, for various numbers $n_{1}, \ldots, \delta_{q}$. Let $C_{i}$ be a general member of $V_{n_{i}, \delta_{i}}^{(0)}$. Then, $C_{1} \cup \cdots \cup C_{q}$ is a curve of degree $n_{1}+\cdots+n_{q}$ with nodes only (i.e., the $C_{i}$ 's meet traversally). 
(3.4) We briefly discuss an obvious generalization, to families, of the classical process of taking a "quadratic transform" of the plane.

Consider a family $X \stackrel{f}{\rightarrow} T, X \subset \mathbf{P}^{2} \times T, T$ integral, of plane curves of degree $d$. Let $t_{0} \in T$ (a closed point), $B=X_{t_{0}} \subset \mathbf{P}^{2} \times\left\{t_{0}\right\} \sim \mathbf{P}^{2}$, and $P_{0}, P_{1}, P_{2}$ points of $\mathbf{P}^{2}$. We shall assume that for some neighborhood $V$ of $t_{0}$, the morphisms $s_{i}: U \rightarrow \mathbf{P}^{2} \times U$ given, on closed points, by $s_{i}(u)=\left(P_{i}, u\right), i=0,1,2$, factor through $X$ (i.e., locally at $t_{0}, s$ admits the trivial sections at $P_{0}, P_{1}, P_{2}$, respectively). Then we may define the standard Cremona transformation of this family. Namely, choose coordinates in $\mathbf{P}^{2}$ so that $P_{0}=(1: 0: 0), P_{1}=(0: 1: 0), P_{2}=(0: 0: 1)$, and use the equations $x_{0}=x_{1}^{\prime} x_{2}^{\prime}$, $x_{1}=x_{0}^{\prime} x_{2}^{\prime}, x_{2}=x_{0}^{\prime} x_{1}^{\prime}$. Precisely, we may find an affine open $U=\operatorname{Spec}(A)$ containing $t_{0}$, such that, over $U$ the restriction $X_{A}$ of $X\left(X_{A} \subset \mathbf{P}_{A}^{2}\right)$ is defined by an equation $F(x)=\sum a_{i j l} x_{0}^{i} x_{i}^{j} x_{2}^{l}=0, i+j+l=n, a_{i j l} \in A$. Then the algebraic transform of $X_{A}$ is given by $F\left(x_{1}^{\prime} x_{2}^{\prime}, x_{0}^{\prime} x_{2}^{\prime}, x_{0}^{\prime} x_{1}^{\prime}\right)=0$. If, for each closed point $t \in U, X_{t}$ has at $P_{i}$ a point of multiplicity $n_{i}$, we may define the proper transform of $X_{A}$ (also referred to as the standard Cremona transformation of $X$ near $t_{0}$ ) to be given in $\mathbf{P}_{A}^{2}$ by $G=0$,

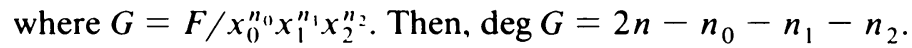

The basic properties of the classical standard Cremona transform for one curve holds for families under the assumption made above. The proofs are essentially the classical ones (see [3, Chapter 7, §4]).

\section{Proof of Theorem (1.4).}

(4.1) Theorem (1.4) will be an easy consequence of Lemmas (4.2) and (4.3) to be given next.

(4.2) Lemмa. Statement (1.4) is true if we make the additional assumption that $n>2 p$.

(4.3) Lemma. Let $B$ be as in (1.4). Choose points $P_{0}, P_{1}, P_{2}$ not in $B$, not on a line, and such that each line determined by two of them cuts $B$ at $n$ distinct points. Consider the standard Cremona transformation of the plane with respect to these points, and the proper transform $B_{1}$ of $B$ (which, in this case, agrees with the algebraic one). Assume that the conclusion of (1.4) holds for $B_{1}$. Then, it also holds for $B$.

(4.4) Proof of (1.4) (accepting The lemmas). It is by induction on the number $b(B)=\inf \left\{b / 2^{b} n>2 p\right\}$ (where $n$ and $p$ are the degree and geometric genus of $B$, respectively). The case $b(B)=0$ is just Lemma (4.2). For the inductive step, if $b(B)=m>0$ and the theorem is valid when $b<m$, take the standard Cremona transformation $B_{1}$ of $B$, which has degree $2 n=n_{1}$, and $2^{m-1} n_{1}=2^{m} n>p$. So, (1.4) is valid for $B_{1}$, and by (4.4) it will be valid for $B$.

(4.5) Proof of Lemma (4.2). Consider, on $B \subset \mathbf{P}^{2}$ (of degree $n$ and geometric genus $p$ ) the linear series $g_{n}^{2}$ cut out by the lines of $\mathbf{P}^{2}$, i.e., by sections in $H^{0}\left(B, \vartheta_{B}(1)\right)$. Take the normalization $q: \bar{B} \rightarrow B$ of $B$, and look at the linear series $\tilde{g}_{n}^{2}$ induced by $g_{n}^{2}$ on $\bar{B}$. If $D \in \tilde{g}_{n}^{2}$, then $D$ is nonspecial (because $\operatorname{deg} D=n>2 p$ by assumption), hence $|D|$ has dimension $n-p$; moreover, since $n>2 p,|D|$ is very ample (see [4, p. 308]). Thus, $|D|$ defines an embedding of $\bar{B}$ into $\mathbf{P}^{n-g}$, i.e., an isomorphism $h: \bar{B} \stackrel{\sim}{\rightarrow} C \subset \mathbf{P}^{n-g}$. More canonically, $\tilde{g}$ corresponds to sections of 
$H^{0}(\bar{B}, \mathcal{E})$, where $\mathcal{E}=q^{*}\left(\vartheta_{B}(1)\right)$. Now we have a natural inclusion

$$
j: H^{0}\left(B, \mathcal{O}_{B}(1)\right) \stackrel{j}{\rightarrow} H^{0}\left(B, q_{*} \mathcal{E}\right) \approx H^{0}(\bar{B}, \mathcal{E}) \quad \text { (because } q \text { is finite) }
$$

$\mathbf{P}^{n-g}$ is $\operatorname{Proj}\left(H^{0}(\bar{B}, \mathcal{E})\right)$, and the composition $g \circ h^{-1}: C \rightarrow B$ is induced from the rational map $\rho: \mathbf{P}^{n-g} \rightarrow P^{2}$ corresponding to $j$. Clearly $\rho$ is the projection (on $\left.L=\operatorname{Proj}\left(\operatorname{Sym} H^{0}\left(B, \Theta_{B},(1)\right)^{*}\right) \sim \mathbf{P}^{2}\right)$ from $S_{0}=\operatorname{Proj}(\operatorname{Sym} K)$, where $K=\operatorname{Ker}\left(j^{*}\right)$ ( $j^{*}$ is the dual of $j$ ). Note: $\operatorname{dim} S_{0}=n-p-3$. Now we let $S_{0}$ vary inside $\mathbf{P}^{n-g}$, i.e., in $\operatorname{Grass}(n-g+1, n-p-2)=G$. If $\mathscr{U}=\{\delta \in G / S \cap L=\varnothing\}$, then for a general element $S$ of $\mathscr{Q}$, the projection of $C$ on $L$ from $S$ will be a nodal curve. In this way we obtain a family (parametrized by $\mathcal{Q}$ ), having the required properties. This proves (4.2).

(4.6) Proof of (4.3). Consider $B_{1}$, the standard Cremona transform of $B$. Since $P_{0}, P_{1}, P_{2}$ are not in $B, B_{1}$ has degree $n_{1}=2 n$ and $g\left(B_{1}\right)=g(B)=p$, because both are birationally equivalent. Moreover, if we write $p=\frac{1}{2}(n-1)(n-2)-d$, then also $p=\frac{1}{2}\left(n_{1}-1\right)\left(n_{1}-2\right)-d_{1}$, with $d_{1}=3 n(n-1) / 2+d$, as a simple calculation shows. We are assuming the following: Using the notation of (3.1), there is an irreducible component $\Sigma_{1}$ of $V_{n_{1}, d_{1}}^{(0)}$ such that $B_{1} \in \Sigma_{1}$ (cf. (3.1)). Now, the singularities of $B_{1}$ are points $Q_{1}, \ldots, Q_{1}$ corresponding to the singularities $S_{1}, \ldots, S_{l}$ of $B$ (via the natural birational correspondence between $B_{1}$ and $B$, which is biregular at those points) and the fundamental points $M_{0}, M_{1}, M_{2}$ of the Cremona transformation (each of the latter is an ordinary $n$-fold point of $B_{1}$ ).

Using the techniques of $\S 3$, we shall construct a subvariety $S$ of $\mathbf{P}^{N_{1}}\left(N_{1}=\right.$ $\frac{1}{2} n_{1}\left(n_{1}+3\right)$, i.e., the space parametrizing curves of degree $\left.n_{1}\right)$, containing $B_{1}$ and having as generic member a curve with $d$ nodes and an $n$-fold point at $M_{i}, i=0,1,2$, such that the Cremona transforms (based at $M_{0}, M_{1}, M_{2}$ ) of the curves in $S$ define the family (1.4) we are looking for. Next we give the details.

Let a general curve $A_{1}$ of $\Sigma_{1}$ (having exactly $d_{1}$ nodes) approach $B_{1}$ in such a way that each of the nodes of $A_{1}$ approaches a singularity of $B_{1}$. We will study how this can happen. (The meaning of these expressions is explained in (3.2), henceforth this terminology will be used without warning.) I claim: If suitably numbered, the nodes of $A_{1}$ can be split into four groups,

$$
\left\{R_{j}^{i}\right\}, \quad i=0,1,2, j=1, \ldots, \frac{1}{2} n(n-1), \quad\left\{R_{j}\right\}, \quad j=1, \ldots, d,
$$

where $R_{j}^{i}$ approaches $M_{i}, i=0,1,2$, and each $R_{j}$ approaches a certain $Q_{i} \in B_{1}(i$ depends on $j$ ). To justify this, recall the meaning of the " $\delta$ invariant" associated to a singular point $P$ of a curve $C, \delta=\lg \left(\overline{\Theta_{C, P}} / \mathcal{O}_{C, P}\right)$. It is the maximum number $m$ such that there will be a family $\phi: Y \rightarrow T$ (T integral), where $\phi^{-1}\left(t_{0}\right)$ is an affine neighborhood $\mathscr{U}$ of $P$ in $C$ such that $\operatorname{Sing}(\mathscr{U})=\{P\}$ and $\phi^{-1}(t)$ has $m$ distinct nodes, and no other singularity (cf. [9, I, §1; II, 5.2]). From this, if we divide the nodes of $A_{1}$ into four groups $\left\{R_{j}^{i}\right\}, i=0,1,2, j=1, \ldots, l_{i},\left\{R_{j}\right\}, j=1, \ldots, l_{3}$, as above, we shall get

$$
l_{i} \leqslant \delta\left(M_{i}, B_{1}\right), \quad i=0,1,2, \quad l_{3} \leqslant \sum_{j=1}^{l} \delta\left(Q_{j}, B_{1}\right) .
$$


But $\delta\left(M_{i}, B_{i}\right)=3 n(n-1) / 2\left(M_{i}\right.$ is an ordinary $n$-fold point $)$ and $\sum_{j=1}^{l} \delta\left(Q_{j}, B_{1}\right)$ $=\Sigma_{j=1}^{\prime} \delta\left(S_{j}, B\right)=d$ by the genus formula. Since $\Sigma_{i-0}^{3} l_{i}=3 n(n-1) / 2+d$ (the total number of nodes of $A_{1}$ ), each of the inequalities above is an equality, which proves our claim.

Now assign the points $R_{1}, \ldots, R_{d}$ on a suitable general $A_{1} \in \Sigma_{1}$. By (3.1)(c), this determines an irreducible component $\Sigma^{\prime}$ of $V_{n_{1}, d}^{(0)}$, in particular $\Sigma_{1} \subset \Sigma^{\prime}$. Note that all these are subvarieties of $\mathbf{P}^{N_{1}}, N_{1}=\frac{1}{2}(2 n(2 n+3))$, and $\operatorname{dim} \Sigma^{\prime}=N_{1}-d$. Now, inside $\mathbf{P}^{N_{1}}$ we have $L_{i}=\left\{D \subset P^{2} / D\right.$ curve of degree $n_{1}$ having an $n$-fold point at $\left.M_{i}\right\}, i=0,1,2$. Each is a linear subvariety of $\mathbf{P}^{N_{1}}$, of dimension $\geqslant \frac{1}{2} n(n+1)$ (this is the number of conditions to get multiplicity $n$ at $\left.M_{i}\right)$. Consider $\left(\cap_{i=0}^{2} L_{i}\right) \cap E^{\prime}$. This contains $B_{1}$, and each irreducible component of it will have dimension

$$
\rho \geqslant 2 n(2 n+3) / 2-d-3 n(n+1) / 2=\left(n^{2}+3 n\right) / 2-d=3 n+p-1 .
$$

Choose an irreducible component $S$ containing $B_{1}$. The universal family on $V_{n_{1}, d}^{(0)}$ (cf. (3.1)(b)) induces a family of plane curves parametrized by $S$, all its members having multiplicity $n$ at $M_{i}, i=0,1,2$. Moreover, the general curve $A^{\prime}$ of $E^{\prime}$ specializes into the general curve $D$ of $S$, which in its turn specializes to $B_{1} \in S$. Since the $d$ nodes of $A^{\prime}$ specialize to $Q_{1}, \ldots, Q_{l}, D$ will have $m \geqslant l$ singularities $F_{1}, \ldots, F_{m}$, distinct from $M_{i}, i=0,1,2$ (and $\delta^{\prime}=\sum_{i=1}^{l} \delta_{i}\left(F_{i}, B\right) \geqslant d$ ), as well as points of multiplicity $\geqslant n$ at $M_{i}, i=0,1,2$. Hence, $\delta\left(M_{i}, D\right) \geqslant n(n-1) / 2$. Hence,

$$
\begin{aligned}
p_{1} & =\text { genus of } D \leqslant(2 n-1)(2 n-2) / 2-d-3 n(n-1) / 2 \\
& =(n-1)(n-2) / 2-d=p .
\end{aligned}
$$

Since $B_{1}$ has genus $p$, and by Theorem (1.2) we have $p \leqslant p_{1}$, we get $p_{1}=p$.

Consider now the standard Cremona transformation of this family based at $M_{0}, M_{1}, M_{2}$. According to (3.4), we obtain a family of plane curves of degree 2, $2 n-3 n=n$, of general genus $p$ (the general curve being birationally equivalent to the previous one), the fiber at $B_{1} \in S$ is $B$. The canonical morphism $\alpha: S \rightarrow \mathbf{P}^{N}$ is clearly injective, hence $T=\overline{\alpha(S)}$ (with the pull-back of the universal curve $\mathscr{D} \subset \mathbf{P}^{2}$ $\times \mathbf{P}^{N}$ ) yields a family of plane curves, having $B$ as a member, $T \subset \mathbf{P}^{N}$, general genus $p$ and $\operatorname{dim} T \geqslant 3 n+p-1$. According to (3.3)(b) (Zariski's Theorem), the general curve of this family is a nodal curve. This proves (3.5).

(4.7) RemarK. A well-known open problem is: Using the notation of (3.1), is it true that there is a single irreducible component of $V_{n, \delta}^{(0)}$ whose general member is an irreducible curve? If the answer is "yes", then Theorem (1.4) would immediately imply: The closure, in $\mathbf{P}^{N}$, of $\{C: C$ is an irreducible plane curve of degree $n$ and genus $p\}$ ( $p$ fixed) is an irreducible algebraic variety.

\section{Proof of Theorem (1.3).}

(5.1) Next we prove Theorem (1.2). In terms of “Severi's varieties" (cf. (3.1)), (1.3) is clearly equivalent to the following statement.

(5.2) Givn a reduced curve $B \subset \mathbf{P}^{2}$ with irreducible components $B_{1}, \ldots, B_{r}$ (we shall write in the sequel $\left.B=B_{1}+\cdots+B_{r}\right)$, where $\operatorname{deg} B_{i}=n_{i}, g\left(B_{i}\right)=p_{i}, i=$ $1, \ldots, r$, and a number $p, p_{1}+\cdots+p_{r} \leqslant p \leqslant(n-1)(n-2) / 2$ (where $n=n_{1}$ $\left.+\cdots+n_{r}\right)$, then there is a number $\delta$ (necessarily equal to $(n-1)(n-2) / 2-p$ ) 
and an irreducible component $\Sigma$ of $V_{n, \delta}^{(0)}(\mathrm{cf}$. (3.1)(a)), $\Sigma$ having as general member an irreducible curve, such that $B \in \Sigma$.

(5.3) We shall prove statement (5.2). We use the notation of (5.2).

By Theorem (1.3), if $\delta_{i}=\left(n_{i}-1\right)\left(n_{i}-2\right) / 2-p_{i}$, then there is an irreducible component $\Sigma_{i}$ of $V_{n_{i}, \delta_{i}}^{(0)}$ such that

$$
B_{i} \subset \Sigma_{i} .
$$

Let $C_{i}$ be a general member of $\Sigma_{i}$ (it will be irreducible, because $B_{i}$ is so). Consider $C=C_{1}+\cdots+C_{r}$. By (3.3)(c), $C$ will be a nodal curve. Let $Q_{1}, \ldots, Q_{\delta^{\prime}}$ be all the nodes of $C$. The degree of $C$ will be $\Sigma_{i=1}^{r} n_{i}=n$. It is easy to see that $B \in V_{n, \delta^{\prime}}^{(())}$(it is easy to construct, using (5.3.1), a family with integral parameter space, showing that $C$ specializes to $B$, then use the universal property of $(3.1)(\mathrm{b})$. Moreover, $C$ is in a single irreducible component of $V_{n, \delta^{\prime}}^{(0)}$, say $\Sigma^{\prime}\left(V_{n, \delta^{\prime}}^{(0)}\right.$ is smooth at $\left.C\right)$.

Now take any integer $h, 0 \leqslant h \leqslant(n-1)(n-2) / 2-\sum_{i=1}^{r} p_{i}$. Clearly, we may choose nodes of $C$, say (after reordering them, if necessary) $Q_{1}, \ldots, Q_{r},{ }_{h}$ forming a "connection set", i.e., $C-\left\{Q_{r+h}, \ldots, Q_{\delta^{\prime}}\right\}$ is connected. Consider the assignment $\left\{Q_{r+h}, \ldots, Q_{\delta^{\prime}}\right\}$ on $C$ (cf. (3.1)(b)). According to (3.1)(b), there is an irreducible component $\Sigma$ of $V_{n, \delta}^{(0)}, \delta=\delta^{\prime}-r-h+1$, whose general member is irreducible, such that $\Sigma$ contains $\Sigma^{\prime}$. In particular, $B \in \Sigma$. We claim: The genus $p$ of the gencral curve of $\Sigma$ is $p_{1}+\cdots+p_{r}+h$. This is a consequence of an elementary calculation, using the fact that $p=(n-1)(n-2) / 2-\delta, \delta=\delta^{\prime}-r-h+1, \delta^{\prime}=\sum_{i=1}^{r} \delta_{i}+$ $\sum_{i<j} n_{i} n_{j}, n=\sum_{i=1}^{r} n_{i}, p_{i}=\left(n_{i}-1\right)\left(n_{i}-2\right) / 2-\delta_{i}$.

Hence, (5.2) has been proved.

\section{BIBLIOGRAPHY}

1. G. Albanese, Sulle condizioni perchè una curva algebraica riducible si possa considerare come limite di una curva irreducibile, Rend. Circ. Mat. Palermo (2) 52 (1928), 105-150.

2. P. Deligne and D. Mumford, The irreducibility of the space of curves of given genus, Inst. Hautes Études Sci. Publ. Math. 15 (1972), 75-110.

3. W. Fulton, Algebraic curves, Benjamin, New York, 1968.

4. R. Hartshorne, Algebraic geometry, Springer-Verlag, New York, 1977.

5. H. Hironaka, On the arithmetic genera and the effective genera of algebraic curves, Mem. Colloq. Univ. Kyoto 30 (1957), 177-195.

6. H. Matsumura, Commutative algebra, Benjamin, New York, 1970.

7. A. Nobile, On families of singular plane projective curves, preprint

8. F. Severi, Vorlesungen über Algebraische Geometrie, Teubner, Leipzig, 1921

9. B. Tessier, Resolution simultanée. I, II, Séminaire sur les Singularités des Surfaces, Lecture Notes in Math., Vol. 777, Springer-Verlag, Berlin and New York, 1980.

10. O. Zariski, Dimension theoretic characterization of maximal irreducible algebraic systems of plane nodal curves of a given order $n$ and with a given number $d$ of nodes, Amer. J. Math. 104 (1982), 209-226.

11. , Contributions to the problem of equisingularity, Questions on Algebraic Varieties, C.I.M.E.

Ed. Cremonese, Roma, 1970, pp. 261-343.

12. Algebraic surfaces, 2nd ed., Springer-Verlag, Heidelberg, 1971.

Department of Mathematics, Louisiana State University, Baton Rouge, Louisiana 70803-4918 